\section{Output Stabilization of Flexible Spacecraft with Active Vibration Suppression}

\author{
S. DI GENNARO \\ Università di L'Aquila \\ Italy
}

Addressed here is the problem of designing a dynamic controller capable of performing rest-to-rest maneuvers for flexible spacecraft, by using attitude measures. This controller does not need the knowledge of modal variables and spacecraft angular velocity. The absence of measurements of these variables is compensated by appropriate dynamics of the controller, which supplies their estimates. The Lyapunov technique is applied in the design of this dynamic controller. Possible source of instability of the controlled system in real cases are the influence of the flexibility on the rigid motion, the presence of disturbances acting on the structure, and parameter variations. In order to attenuate their effects and to damp out undesirable vibrations affecting the spacecraft attitude, distributed piezoelectric actuators are used. In fact, in presence of disturbances and/or parameter variation the proposed controller ensures an approximate solution of the control problem.

Manuscript received October 30, 1999; revised April 5, 2002; released for publication April 1, 2003.

IEEE Log No. T-AES/39/3/818479.

Refereeing of this contribution was handled by T. F. Roome.

This research was partially supported by the "Agenzia Spaziale Italiana" and by the University of L'Aquila, "Progetto di Ateneo-Il controllo attivo nella meccanica delle strutture."

Author's address: Dipartimento di Ingegneria Elettrica, Università di L'Aquila, 67040 Poggio di Roio, L'Aquila, Italy, E-mail: (digennar@ing.univaq.it).

$\overline{0018-9251 / 03 / \$ 17.00 ~(c) ~} 2003$ IEEE

\section{INTRODUCTION}

A desired characteristic of future spacecraft and space structures is the possibility of reducing the costs inherent with their transport in the space. Hence, it is necessary to decrease their weight; the main drawback consists of the decreased rigidity of the structure. Therefore, the elimination of vibrations becomes an important issue in spacecraft maneuvers.

Another issue, related to the previous one, is the reduction of the number of sensors necessary to implement a control strategy. This is an interesting aspect in case of sensor failures and for the reduction of the structure and control system design costs. In this case the main problem is the measure of the variables describing the flexible motion, the modal position, and velocity. Additional problems arise when one measures only the output, namely some variables are not available for measuring, such as the spacecraft angular velocity. The effects of the flexible dynamics, affecting the rigid motion, and the absence of measurements of the corresponding modal variables and of the angular velocity, render the controller task more severe. In fact, the reduced accuracy in the control action, due to the use of estimates, may impair the stability of the overall control scheme.

An interesting solution for the attenuation of the flexible oscillations induced by spacecraft maneuvers is given by piezoelectric actuators [1-6]. These actuators consist of films of piezoelectric material placed along the flexible elements of the structure. Due to their inherent distributed nature, the piezoelectric actuators are natural candidates for damping out undesirable vibrations.

The techniques present in the literature, among which the nonlinear input-output decoupling and linearization [7-14], fail to give an appropriate answer to the active control problem from output measurements, since they need the knowledge of the entire state of the system. Hence, for solving this problem, in this work a dynamic controlled is proposed, based on the Lyapunov technique (see for instance [15] for its application in the design of spacecraft control systems). The controller derived uses estimates of the modal variables and of the angular velocity. Since the total angular momentum remains constant when external torques are absent, the estimate of the spacecraft angular velocity is easily determined; a similar approach can be used when the external torque depends on known variables. When disturbances are absent, see also [16] for a recent result on attitude control from quaternion measures. In the presence of disturbance torques and when we deal with system parameter variations, the same control scheme fails to solve exactly the posed control problem. In this case and under appropriate conditions on the disturbances and parameter variations, it is possible to show that 
the problem can be solved from a practical point of view, namely it is ensured that the norm of the system state can be rendered smaller than a certain constant, depending on the size of the perturbation. In both cases the vibrations due to rapid maneuvers are damped out by piezoelectric films bonded to the flexible structures. The basic action of the piezoelectric actuators is to increase the stiffness and the internal damping, so reducing the time necessary to damp out the vibrations induced by large and rapid maneuvers.

It is worth noting that the same approach can be generalized to the case of attitude tracking, following the method used in [17], where active actuators were used and dynamic controllers were designed under the further hypothesis of spacecraft angular rate measure. Since the mathematics become more complex, and for the sake of simplicity, we restrict our attention to the simpler case of rest-to-rest maneuvers.

This article is organized as follows. In Section II the mathematical model of a flexible spacecraft with piezoelectric actuators is recalled, and the control problem is formulated. In Section III a dynamic controller is designed and its validity is analyzed in the case of disturbances and/or parameter variations. Simulation results and comparisons are shown in Section IV. Some comments conclude the paper.

\section{MATHEMATICAL MODEL AND PROBLEM STATEMENT}

\section{A. Mathematical Model of a Flexible Spacecraft}

The flexible spacecraft is composed of a rigid main body and some flexible appendages. The kinematics of the spacecraft determine the attitude of the main body, and are described by the four unitary quaternions [7-10, 18, 19]

$$
q_{0}=\cos \frac{\Phi}{2}, \quad q=\epsilon \sin \frac{\Phi}{2}
$$

subject to the constraint $q_{0}^{2}+q^{T} q=1$. Here $\Phi$ denotes the rotation angle about the Euler axis, which is determined by the unitary vector $\epsilon$. Then the kinematic equations take the form

$$
\left(\begin{array}{c}
\dot{q}_{0} \\
\dot{q}
\end{array}\right)=\frac{1}{2} \mathcal{Q}\left(q_{0}, q\right) \omega
$$

where $\omega$ is the angular velocity of the undeformed spacecraft in the body fixed frame, and

$$
\mathcal{Q}\left(q_{0}, q\right)=\left(\begin{array}{c}
-q^{T} \\
q_{0} I+\tilde{q}
\end{array}\right)
$$

with $I$ the identity matrix and $\tilde{q}$ the dyadic representation of $q$ [19].
The dynamic equations of a spacecraft with flexible appendages, actuated by gas jets and/or reaction wheels, can be found in [6-11] and are given by

$$
\begin{aligned}
J \dot{\omega}+\delta^{T} \ddot{\eta} & =-\tilde{\omega}\left(J \omega+\delta^{T} \dot{\eta}\right)+u+D \\
\ddot{\eta}+C \dot{\eta}+K \eta & =-\delta \dot{\omega}-\delta_{2} u_{p} .
\end{aligned}
$$

Equation (2) is given by Euler theorem [19] and describes, in the body-fixed frame, the rigid dynamics of the total angular momentum, given by

$$
\chi=J \omega+\delta^{T} \dot{\eta} .
$$

In this equation $J$ is the symmetric inertia matrix of the whole structure, which is the sum of the symmetric inertia matrix $J_{m b}$ of the main body (which is clearly a positive definite matrix) and a symmetric inertia matrix due to the flexible structure (whose form is specified in the following). Moreover, $\delta$ is the coupling matrix between the elastic and rigid structures, $\eta$ is the modal coordinate vector, $u$ is the external torque. It is worth noting that this torque can be generated either by gas jets or by reaction wheels; in this second case, if $\dot{\Omega}$ denotes the reaction wheel angular acceleration, the imposed torque is given by $u=J_{r} \dot{\Omega}$, where $J_{r}$ is the reaction wheel inertia matrix (torque-shaping). $D$ is a term taking into account external disturbance torques and possible variations with respect to the parameter nominal values (see for instance [20]), present in practical situations; therefore, in general $D$ depends explicitly on $t$ and on the state of the system. Here $\tilde{\omega}$ is the dyadic representation of $\omega$. Equation (3) describes the flexible dynamics, under the hypothesis of small elastic deformations; $C=\operatorname{diag}\left\{2 \zeta_{i} \omega_{n i}, i=1, \ldots, N\right\}$, $K=\operatorname{diag}\left\{\omega_{n i}^{2}, i=1, \ldots, N\right\}$ are the damping and stiffness matrices, $N$ is the number of elastic modes considered, $\omega_{n i}$ are the natural frequencies, and $\zeta_{i}$ the corresponding dampings. Since on the surface of the flexible appendages thin, homogeneous, and isotropic films of piezoelectric actuators are bounded, in (3) the piezoelectric input $u_{p}$ appears, which influences the flexible dynamics through the coupling matrix $\delta_{2}$. Note that the term $\delta_{2} u_{p}$ results from the integration over the length of the flexible appendage of the contribution to the bending moment of the voltage input $u_{p}$, time-varying but constant along the piezoelectric film. The bending moments, due to the piezoelectric films, around the neutral axis of the composite boom have the expression

$$
M_{p}=-c_{p} u_{p}
$$

with $c_{p}$ a constant depending on the geometry and the nature of materials [1-3]. Finally, note that $u$ influence the flexible dynamics (3) through the term $\delta \dot{\omega}$, while $u_{p}$ influences the rigid ones (2) by means of $\delta^{T} \ddot{\eta}$. 
From (1)-(3) one obtains the dynamics of a flexible spacecraft with piezoelectric actuators

$$
\begin{aligned}
\left(\begin{array}{c}
\dot{q}_{0} \\
\dot{q}
\end{array}\right) & =\frac{1}{2} \mathcal{Q}\left(q_{0}, q\right) \omega \\
\dot{\omega}= & J_{m b}^{-1}\left[-\tilde{\omega}\left(J \omega+\delta^{T} \dot{\eta}\right)+\delta^{T}(K \eta+C \dot{\eta})\right. \\
& \left.\quad+u+\delta_{1} u_{p}+D\right] \\
\ddot{\eta} & =-\delta \dot{\omega}-(K \eta+C \dot{\eta})-\delta_{2} u_{p}
\end{aligned}
$$

where one sets $\delta_{1}=\delta^{T} \delta_{2}$ and $J=J_{m b}+\delta^{T} \delta$, with $\delta^{T} \delta$ is the contribution of the flexible parts to the total inertia matrix.

\section{B. Problem Statement}

In this work we consider rest-to-rest maneuvers; the control aim is to realize desired rotations and, at the same time, to damp out the vibrations induced by these maneuvers in the flexible elements of the spacecraft, in presence of disturbances and/or parameter variations. The measured variables are the attitude variables $q_{0}, q$. This problem can be summarized as follows. Find a controller such that, using the measurements $q_{0}, q$,

$$
\lim _{t \rightarrow \infty} q=0, \quad \lim _{t \rightarrow \infty} \eta=0, \quad \lim _{t \rightarrow \infty} \dot{\eta}=0 .
$$

It is worth noting that when $q \rightarrow 0$, we have $q_{0} \rightarrow 1$, because of the constraint relation.

The attitude rotations can be commanded by the gas jets and/or by the reaction wheels. The piezoelectric actuators are used to actively damp out the vibrations induced by these maneuvers. Since the aim is to design a controller which uses only attitude measurements, what we need are information regarding the modal variable $\eta$ and the spacecraft angular velocity $\omega$. As we will see, these information can be obtained by designing, in the controller, a dynamic system whose outputs are the estimates of $\eta, \dot{\eta}, \omega$, while the inputs are $q_{0}, q$.

At this point a brief discussion deserves the knowledge of the total angular momentum (4). We suppose that at the initial time the spacecraft is idle, namely the total angular momentum at $t=0$ is zero, $\chi(0)=0$. Unfortunately, we do not know its value at $t>0$. In fact, in its expression the angular velocity $\omega$ and the modal velocity $\dot{\eta}$ are unknown. Hence, in general $\chi$ cannot be considered known. Nevertheless, in particular cases, namely when internal actuators are used (e.g. piezoelectric actuators), the applied control $u$ depends on known quantities $\left(q_{0}\right.$ and $\left.q\right)$, and no disturbances act on the spacecraft, then $\chi$ can be considered known [11]. In fact, a vector expressed in the body-fixed frame can be reexpressed in the inertial frame by using the orthonormal matrix [19]

$$
\mathcal{R}_{i b}\left(q_{0}, q\right)=I+2\left(q_{0} I+\tilde{q}\right) \tilde{q}, \quad \mathcal{R}_{i b}^{T}\left(q_{0}, q\right)=\mathcal{R}_{b i}\left(q_{0}, q\right)
$$

depending on the attitude quaternions. If $\chi^{i}, \chi$ denote the total angular momentum expressed in the inertial frame and in the body-fixed frame, then $\chi^{i}=\mathcal{R}_{i b}\left(q_{0}, q\right) \chi$. Now in the inertial frame (2) is written as $(d / d t) \chi^{i}=u^{i}=\mathcal{R}_{i b}\left(q_{0}, q\right) u$, where $d / d t$ and $u^{i}$ denote the time derivative and the external torque, both expressed in the inertial frame; hence

$$
\chi^{i}=\int_{0}^{t} \mathcal{R}_{i b}\left(q_{0}(\tau), q(\tau)\right) u(\tau) d \tau .
$$

Therefore

$$
\begin{array}{r}
\chi=\chi_{u}:=\mathcal{R}_{b i}\left(q_{0}, q\right) \int_{0}^{t} \mathcal{R}_{i b}\left(q_{0}(\tau), q(\tau)\right) u(\tau) d \tau, \\
\chi(0)=0
\end{array}
$$

can be considered known. As already noted, at $t=0$ the total angular momentum is zero since rest-to-rest maneuvers are considered. It is also worth noting that if the control $u$ is implemented by gas jet actuators, which behave in a "bang-bang" mode, the calculation of the the integral in (6) is easier.

If a disturbance is acting on the system or parameter variations must be considered, as in a realistic situation, one has to take into account the following unknown term

$$
\chi_{D}:=\mathcal{R}_{b i}\left(q_{0}, q\right) \int_{0}^{t} \mathcal{R}_{i b}\left(q_{0}(\tau), q(\tau)\right) D(\tau) d \tau
$$

which is the contribution to $\chi$ due to disturbances and/or parameter variations affecting the system. Hence, in this case the expression of the total angular momentum is

$$
\chi=\chi_{u}+\chi_{D}
$$

and clearly $\chi$ cannot be considered known. Note that for generic disturbances, the total angular momentum could increase (theoretically) with no bounds as the time grows, and in absence of angular velocity measurements the control problem would have no solution. Therefore, we need to impose some hypothesis on $D$. As shown later on, when $D$ is bounded the control problem can be solved in an approximated way, at least for finite time intervals.

\section{DYNAMIC CONTROLLER BASED ON ATTITUDE MEASUREMENTS}

In this section a dynamic controller, based on attitude measures, is derived making use of the standard Lyapunov technique. Estimates of the modal variables are obtained by using dynamics which reproduce the simple linear dynamics governing the flexible motion. The modal estimate dynamics contain terms which take into account the coupling between rigid and flexible motion. The estimate of the modal variables from measurements of $q_{0}, q$ is possible since the rigid dynamics are influenced by the flexible ones 
through the coupling matrix $\delta$. The design of this controller follows the approach used in [6], where the angular velocity is supposed measurable, and exploits ideas contained in [21]. We first consider the case in which $D=0$ in (5); in this case the control problem can be solved in an exact way.

We introduce first some notations. Let $R$ a fixed symmetric positive definite matrix. In what follows it is necessary to determine the solution $P$ of the following Lyapunov equation

$$
\left[P\left(\begin{array}{cc}
0 & I \\
-\tilde{K} & -\tilde{C}
\end{array}\right)+\left(\begin{array}{cc}
0 & I \\
-\tilde{K} & -\tilde{C}
\end{array}\right)^{T} P\right] / 2=-R
$$

where $\tilde{K}=K+\delta_{2} F_{1}, \tilde{C}=C+\delta_{2} F_{2}$ are the stiffness and damping matrices of the elastic structure determined by the piezoelectric actuator; $F_{1}$ and $F_{2}$ are appropriate matrices introduced later on in the expression of the piezoelectric input $u_{p}$.

Let us consider a partition of the matrix $P$ in matrices $N \times N$

$$
P=\left(\begin{array}{cc}
P_{1} & P_{2} \\
P_{2}^{T} & P_{3}
\end{array}\right)
$$

and define the matrices

$$
\begin{aligned}
& A_{11}=F_{1}^{T} \delta_{2}^{T} P_{2} \\
& A_{12}=F_{1}^{T} \delta_{2}^{T} P_{3}+\left(P_{1}-P_{2} C-K\right) \delta J_{m b}^{-1} \delta^{T} \\
& A_{21}=F_{2}^{T} \delta_{2}^{T} P_{2} \\
& A_{22}=F_{2}^{T} \delta_{2}^{T} P_{3}+\left[\left(P_{2}^{T}-P_{3} C-C\right) \delta+k_{d} \delta J_{m b}^{-1}\right] J_{m b}^{-1} \delta^{T}
\end{aligned}
$$

where $k_{d}$ is a positive gain introduced in the control $u$ later on. Let us consider now a symmetric positive definite matrix $\bar{Q}$ such that the matrix

$$
Q=\bar{Q}-\left(\begin{array}{ll}
A_{11} & A_{12} \\
A_{21} & A_{22}
\end{array}\right)
$$

is positive definite. Moreover, let $\Gamma$ be the solution of the following Lyapunov equation

$$
\left[\Gamma\left(\begin{array}{cc}
0 & I_{0} \\
-K & -C_{0}
\end{array}\right)+\left(\begin{array}{cc}
0 & I_{0} \\
-K & -C_{0}
\end{array}\right)^{T} \Gamma\right] / 2=-\bar{Q}
$$

with

$$
I_{0}=I+\delta J_{m b}^{-1} \delta^{T}, \quad C_{0}=C I_{0} .
$$

Note that $I_{0}$ is positive definite and that the matrix

$$
\left(\begin{array}{cc}
0 & I_{0} \\
-K & -C_{0}
\end{array}\right)=\left(\begin{array}{cc}
0 & I \\
-K & -C
\end{array}\right)\left(\begin{array}{cc}
I & 0 \\
0 & I_{0}
\end{array}\right)
$$

has eigenvalues with negative real parts. In fact, it is the product of a matrix (the first on the right side) with negative eigenvalues (recall that $K$ and $C$ are diagonal positive definite matrices) with a matrix (the second on the right side) which is positive definite. Therefore, there exists a symmetric positive definite matrix $\Gamma$ such that (12) is verified. Now we are ready to state our first result.

THEOREM 1 Let us consider the unperturbed system (5) with $D=0$. Let us suppose that the measured variables are the quaternions $q_{0}, q$. Let

$$
\hat{\omega}=J_{m b}^{-1}\left(\chi-\delta^{T} \hat{\psi}\right)
$$

be the estimate of the angular velocity $\omega$, where $\chi$ is given by (6). Moreover, let $F_{1}, F_{2}$ be two matrices such that the matrix

$$
\left(\begin{array}{cc}
0 & I \\
-\left(K+\delta_{2} F_{1}\right) & -\left(C+\delta_{2} F_{2}\right)
\end{array}\right)
$$

is Hurwitz. The following dynamic controller

$$
\begin{aligned}
\left(\begin{array}{c}
\dot{\hat{\eta}} \\
\dot{\hat{\psi}}
\end{array}\right)= & \left(\begin{array}{c}
\hat{\psi}-\delta \hat{\omega} \\
-C \hat{\psi}-K \hat{\eta}+C \delta \hat{\omega}-\delta_{2}\left(F_{1} \hat{\eta}+F_{2} \hat{\psi}\right)
\end{array}\right) \\
& +\Gamma^{-1}\left[\left(\begin{array}{c}
K \delta \\
C \delta-k_{d} \delta J_{m b}^{-1}
\end{array}\right) \hat{\omega}+P\left(\begin{array}{c}
-I \\
C
\end{array}\right) \delta \hat{\omega}\right. \\
& \left.+\left(\begin{array}{cc}
0 & F_{1}^{T} \delta_{2}^{T} \\
0 & F_{2}^{T} \delta_{2}^{T}
\end{array}\right) P\left(\begin{array}{c}
\hat{\eta} \\
\hat{\psi}
\end{array}\right)\right] \\
u= & -k_{p} q-k_{d} \hat{\omega}-\delta^{T} \\
& \times\left[\begin{array}{lll}
K & C
\end{array}\right)+\left(\begin{array}{ll}
-I & C) P+\delta_{2}\left(F_{1}\right. \\
\left.F_{2}\right)
\end{array}\right)\left(\begin{array}{c}
\hat{\eta} \\
\hat{\psi}
\end{array}\right) \\
u_{p}= & F_{1} \hat{\eta}+F_{2} \hat{\psi}
\end{aligned}
$$

where $k_{p}, k_{d}>0$ and $P, \Gamma$ are the solutions of (9) and (12) respectively, solves the control problem. Finally, $\hat{\eta}$ is the estimate of $\eta$ and $\hat{\psi}-\delta \hat{\omega}$ is the estimate of $\dot{\eta}$.

REMARK 1 The matrices $F_{1}, F_{2}$ are designed so that the eigenvalues of the controllable flexible dynamics are the desired ones. To see this, note first that the control $u_{p}$ can be rewritten as

$$
u_{p}=F_{1} \eta+F_{2} \psi-F_{1}(\eta-\hat{\eta})-F_{2}(\psi-\hat{\psi}) .
$$

If the controller dynamics are such that the errors $(\eta-\hat{\eta}),(\psi-\hat{\psi})$ tend asymptotically to zero, the controllable eigenvalues of the flexible dynamics remain fixed. In fact, the flexible dynamics are

$$
\begin{aligned}
\ddot{\eta}= & -\left(K+\delta_{2} F_{1}\right) \eta-\left(C+\delta_{2} F_{2}\right) \dot{\eta}-\delta \dot{\omega}-\delta_{2} F_{2} \delta \omega \\
& +\delta_{2} F_{1}(\eta-\hat{\eta})+\delta_{2} F_{2}(\psi-\hat{\psi})
\end{aligned}
$$

and when the maneuver is completed, i.e. $\omega=0$, $\dot{\omega}=0$, and if the errors $(\eta-\hat{\eta}),(\psi-\hat{\psi})$ are zero, these dynamics become

$$
\ddot{\eta}=-\left(K+\delta_{2} F_{1}\right) \eta-\left(C+\delta_{2} F_{2}\right) \dot{\eta} .
$$


From this it is clear that the role of the active actuators is to increase the structure damping and stiffness.

Let us prove Theorem 1. First, we introduce the following variable

$$
\psi=\dot{\eta}+\delta \omega
$$

representing the total angular velocity expressed in modal variables [9]. It simplifies the following developments. Note that

$$
\dot{\psi}=-C \dot{\eta}-K \eta-\delta_{2} u_{p}=-C \psi-K \eta+C \delta \omega-\delta_{2} u_{p} .
$$

Then, we consider the following continuously differentiable function

$$
\begin{aligned}
V= & k_{p}\left[\left(q_{0}-1\right)^{2}+q^{T} q\right]+\frac{1}{2} \omega^{T} J_{m b} \omega+\frac{1}{2}\left(\eta^{T} \quad \psi^{T}\right) P\left(\begin{array}{c}
\eta \\
\psi
\end{array}\right) \\
& +\frac{1}{2}\left(\begin{array}{ll}
e_{\eta}^{T} & \left.e_{\psi}^{T}\right) \Gamma\left(\begin{array}{c}
e_{\eta} \\
e_{\psi}
\end{array}\right) .
\end{array}\right.
\end{aligned}
$$

Here $P, \Gamma$ are symmetric positive definite matrices to be determined as in (9), (12), and

$$
e_{\eta}=\eta-\hat{\eta}, \quad e_{\psi}=\psi-\hat{\psi} .
$$

Computing the derivative of $V$ and taking into account the dynamics (5) with $D=0$, one obtains

$$
\begin{aligned}
& \dot{V}=k_{p} \omega^{T} q+\omega^{T}\left[-\tilde{\omega} \chi+\delta^{T}(C \psi+K \eta-C \delta \omega)+u+\delta_{1} u_{p}\right] \\
& +\left(\begin{array}{ll}
\eta^{T} & \psi^{T}
\end{array}\right) P\left(\begin{array}{c}
\psi-\delta \omega \\
-C \psi-K \eta+C \delta \omega-\delta_{2} u_{p}
\end{array}\right) \\
& +\left(\begin{array}{ll}
e_{\eta}^{T} & e_{\psi}^{T}
\end{array}\right) \Gamma\left(\begin{array}{c}
\dot{e}_{\eta} \\
\dot{e}_{\psi}
\end{array}\right) .
\end{aligned}
$$

Note that this expression can be simplified since $\omega^{T} \tilde{\omega} \chi=0$. From (4) one works out

$$
\omega=J_{m b}^{-1}\left(\chi-\delta^{T} \psi\right) .
$$

Note that due to the definition of $J, J_{m b}$ is a positive definite matrix. Therefore, one can introduce the angular rate estimate (14). From (21) and (14)

$$
e_{\omega}=-J_{m b}^{-1} \delta^{T} e_{\psi}
$$

with $e_{\omega}=\omega-\hat{\omega}$. With the control (17) the derivative (20) assumes the form

$$
\begin{aligned}
\dot{V}= & -\omega^{T}\left(k_{d} I+\delta^{T} C \delta\right) \omega-\left(\begin{array}{ll}
\eta^{T} & \psi^{T}
\end{array}\right) R\left(\begin{array}{c}
\eta \\
\psi
\end{array}\right) \\
& +\left(\begin{array}{cc}
e_{\eta}^{T} & e_{\psi}^{T}
\end{array}\right)\left[\Gamma\left(\begin{array}{c}
\dot{e}_{\eta} \\
\dot{e}_{\psi}
\end{array}\right)+\left(\begin{array}{c}
K \delta \\
C \delta-k_{d} \delta J_{m b}^{-1}
\end{array}\right) \omega\right. \\
& \left.+P\left(\begin{array}{c}
-I \\
C
\end{array}\right) \delta \omega+\left(\begin{array}{ll}
0 & F_{1}^{T} \delta_{2}^{T} \\
0 & F_{2}^{T} \delta_{2}^{T}
\end{array}\right) P\left(\begin{array}{c}
\eta \\
\psi
\end{array}\right)\right]
\end{aligned}
$$

where $R=R^{T}>0$ is fixed, so that $P$ is calculated from (9). The last term in (23) can be expanded according to (5) and (18)

$$
\begin{aligned}
\left(\begin{array}{cc}
e_{\eta}^{T} & e_{\psi}^{T}
\end{array}\right)[ & P\left(\begin{array}{c}
\psi-\delta \omega-\dot{\hat{\eta}} \\
-C \psi-K \eta+C \delta \omega-\delta_{2}\left(F_{1} \hat{\eta}+F_{2} \hat{\psi}\right)-\dot{\hat{\psi}}
\end{array}\right) \\
& +\left(\begin{array}{c}
K \delta \\
C \delta-k_{d} \delta J_{m b}^{-1}
\end{array}\right) \omega+P\left(\begin{array}{c}
-I \\
C
\end{array}\right) \delta \omega \\
& \left.+\left(\begin{array}{cc}
0 & F_{1}^{T} \delta_{2}^{T} \\
0 & F_{2}^{T} \delta_{2}^{T}
\end{array}\right) P\left(\begin{array}{c}
\eta \\
\psi
\end{array}\right)\right] .
\end{aligned}
$$

Choosing the estimate dynamics (16) one finally has

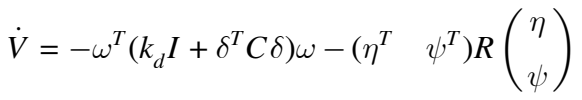

$$
\begin{aligned}
& +\left(\begin{array}{ll}
e_{\eta}^{T} & e_{\psi}^{T}
\end{array}\right)\left[\Gamma\left(\begin{array}{cc}
0 & I_{0} \\
-K & -C_{0}
\end{array}\right)+\left(\begin{array}{ll}
A_{11} & A_{12} \\
A_{21} & A_{22}
\end{array}\right)\right]\left(\begin{array}{l}
e_{\eta} \\
e_{\psi}
\end{array}\right)
\end{aligned}
$$

where $A_{i j}$ are given by (10). Fixing $Q$ as in (11), so that $\Gamma$ remains determined by (12), one obtains

$$
\begin{aligned}
\dot{V}= & -\omega^{T}\left(k_{d} I+\delta^{T} C \delta\right) \omega-\left(\begin{array}{ll}
\eta^{T} & \psi^{T}
\end{array}\right) R\left(\begin{array}{l}
\eta \\
\psi
\end{array}\right) \\
& -\left(\begin{array}{ll}
e_{\eta}^{T} & e_{\psi}^{T}
\end{array}\right) Q\left(\begin{array}{c}
e_{\eta} \\
e_{\psi}
\end{array}\right) \leq 0 .
\end{aligned}
$$

Let $E$ be the set of points where $\dot{V}=0$, and let us indicate by $\mathcal{E}$ the largest invariant set in $E$ [22]. It is clear that $E$ is constituted by the points

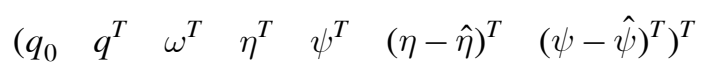

such that

$$
\omega=0, \quad \eta=0, \quad \psi=0, \quad \hat{\eta}=\eta, \quad \hat{\psi}=\psi .
$$

From (18) and (22), this implies

$$
\dot{\eta}=0, \quad \hat{\omega}=\omega .
$$

In order to determine $\mathcal{E}$, note that from (5) and (17)

$$
\dot{\omega}=0=-J_{m b}^{-1} k_{p} q
$$

i.e., $q=0$. On the basis of La Salle theorem [22], every solution of the controlled system (5), (16), (17) approaches $\mathcal{E}$ as $t \rightarrow \infty$. Therefore, the dynamic controller (16), (17) solves the control problem. This completes the proof of Theorem 1.

REMARK 2 Note that the control law $u$ can be rewritten in term of estimate errors

$$
\begin{aligned}
u= & -k_{p} q-k_{d} \omega-\delta_{1} u_{p}+\delta^{T} \delta_{2} F_{1} \eta-2 \delta^{T} C \psi-\delta^{T} \delta_{2} F_{1} e_{\eta} \\
& +\left(2 \delta^{T} C-k_{d} J_{m b}^{-1} \delta^{T}\right) e_{\psi} .
\end{aligned}
$$

As $e_{\eta}, e_{\psi} \rightarrow 0, u$ assumes the expression of the stabilizing control law for a flexible spacecraft in case of whole state measure.

REMARK 3 Using arguments similar to those presented in [6], the extension of the previous results 
to the case of attitude tracking is not difficult, even if mathematically more complex. Moreover, it is not difficult to include in the control the term which (asymptotically) cancels the gyroscopic term, in order to obtain a better performance.

REMARK 4 It is easily seen that the controller (16), (17) simplifies if the $3 \times 3$ matrix $\delta^{T} C \delta$ has full rank. In fact, in this case it is possible to set $k_{d}$ to zero, that is the controller does not contain the "derivative" term, expressed by the estimate of $\omega$

$$
\begin{aligned}
& \left(\begin{array}{c}
\dot{\hat{\eta}} \\
\dot{\hat{\psi}}
\end{array}\right)=\left(\begin{array}{c}
\hat{\psi}-\delta \hat{\omega} \\
-C \hat{\psi}-K \hat{\eta}+C \delta \hat{\omega}-\delta_{2}\left(F_{1} \hat{\eta}+F_{2} \hat{\psi}\right)
\end{array}\right) \\
& +\Gamma^{-1}\left[\left(\begin{array}{l}
K \\
C
\end{array}\right) \delta \hat{\omega}+P\left(\begin{array}{c}
-I \\
C
\end{array}\right) \delta \hat{\omega}+\left(\begin{array}{ll}
0 & F_{1}^{T} \delta_{2}^{T} \\
0 & F_{2}^{T} \delta_{2}^{T}
\end{array}\right) P\left(\begin{array}{l}
\hat{\eta} \\
\hat{\psi}
\end{array}\right)\right] \\
& \left.u=-k_{p} q-\delta^{T}\left[\begin{array}{lll}
K & C
\end{array}\right)+\left(\begin{array}{lll}
-I & C
\end{array}\right) P+\delta_{2}\left(F_{1} \quad F_{2}\right)\right]\left(\begin{array}{l}
\hat{\eta} \\
\hat{\psi}
\end{array}\right) \\
& u_{p}=F_{1} \hat{\eta}+F_{2} \hat{\psi}
\end{aligned}
$$

and

$$
\begin{aligned}
& \dot{V}=-\omega^{T} \delta^{T} C \delta \omega-\left(\begin{array}{ll}
\eta^{T} & \psi^{T}
\end{array}\right) R\left(\begin{array}{l}
\eta \\
\psi
\end{array}\right) \\
& +\left(\begin{array}{ll}
e_{\eta}^{T} & e_{\psi}^{T}
\end{array}\right)\left[\Gamma\left(\begin{array}{cc}
0 & I_{0} \\
-K & -C_{0}
\end{array}\right)+\left(\begin{array}{ll}
A_{11} & A_{12} \\
A_{21} & \bar{A}_{22}
\end{array}\right)\right]\left(\begin{array}{c}
e_{\eta} \\
e_{\psi}
\end{array}\right)
\end{aligned}
$$

with

$$
\bar{A}_{22}=F_{2}^{T} \delta_{2}^{T} P_{3}+\left(P_{2}^{T}-P_{3} C-C\right) \delta J_{m b}^{-1} \delta^{T}
$$

and $A_{11}, A_{12}, A_{21}$ as in (10). By applying La Salle theorem, we obtain again that $q \rightarrow 0$. Note that in the general case, when $\delta^{T} C \delta$ is not a full rank matrix, we cannot arrive at the same conclusions since it is not true that neither $\omega \rightarrow 0$ nor $\dot{\omega} \rightarrow 0$.

The fact that $u$ does not contain the term proportional to $\hat{\omega}$ is possible since the information about the angular velocity are contained in $\psi$ through the estimate $\hat{\psi}$.

Let us now investigate the effect of the controller presented in Theorem 1 on the real system (5), where the term $D$, accounting for disturbances and/or parameter variations is now present. As previously noted, for generic disturbances the total angular momentum could increase as the time grows, according to (7). Hence, it is obvious to consider bounded disturbances. But even in this case it is clear from (7) that $\chi_{D} \rightarrow \infty$ as $t \rightarrow \infty$. Therefore, unless to consider integrable disturbances, we will be able to solve (approximately) the control problem only for finite time intervals. This is physically clear: since the disturbance $D$ modifies the total angular momentum $\chi$, and therefore the angular velocity $\omega$ (which is not measured), it is necessary that the "action" of $D$ is limited (if not in amplitude, at least in time) in order to hope to obtain an appropriate estimate of $\omega$.
The following result shows that if $D$ is bounded the controller of Theorem 1 solves the control problem in the sense of ultimate boundedness of the trajectories [22] at least for finite time intervals, when disturbances and/or parameter variations are present.

THEOREM 2 Let us consider the perturbed system (5) with $D$ such that $\|D\| \leq \alpha_{1}$. The controller (16), (17) of Theorem 1 ensures the existence of a time instant $T>0$ and an appropriate bound $b>0$ such that $\left\|\left(1-q_{0}\right) q^{T} \eta^{T} \psi^{T} e_{\eta}^{T} e_{\psi}^{T}\right\| \leq b$ at least for $t \leq T$.

Let us prove Theorem 2. We use the same symbology introduced for Theorem 1 . We consider again the function (19) as Lyapunov function candidate $V$. Computing the derivative of $V$ along the system dynamics (5), one has

$$
\begin{aligned}
& \dot{V}=k_{p} \omega^{T} q+\omega^{T}\left[-\tilde{\omega} \chi+\delta^{T}(C \psi+K \eta-C \delta \omega)+u+\delta_{1} u_{p}+D\right]
\end{aligned}
$$

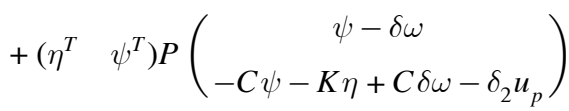

$$
\begin{aligned}
& +\left(\begin{array}{ll}
e_{\eta}^{T} & e_{\psi}^{T}
\end{array}\right) \Gamma\left(\begin{array}{c}
\dot{e}_{\eta} \\
\dot{e}_{\psi}
\end{array}\right) .
\end{aligned}
$$

Note in this expression the presence of the term $\omega^{T} D$. Moreover, note that (4), (8) yield

$$
\omega=J_{m b}^{-1}\left(\chi_{u}+\chi_{D}-\delta^{T} \psi\right)
$$

so that, from (14)

$$
e_{\omega}=-J_{m b}^{-1} \delta^{T} e_{\psi}+J_{m b}^{-1} \chi_{D} .
$$

Therefore, with the control (17) the derivative $\dot{V}$ assumes now the form

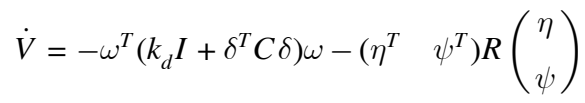

$$
\begin{aligned}
& +\omega^{T} D+k_{d} \omega^{T} J_{m b}^{-1} \chi_{D}+\left(\begin{array}{ll}
e_{\eta}^{T} & e_{\psi}^{T}
\end{array}\right) \\
& \times\left[\Gamma\left(\begin{array}{c}
\dot{e}_{\eta} \\
\dot{e}_{\psi}
\end{array}\right)+\left(\begin{array}{c}
K \delta \\
C \delta-k_{d} \delta J_{m b}^{-1}
\end{array}\right) \omega\right. \\
& \left.+P\left(\begin{array}{c}
-I \\
C
\end{array}\right) \delta \omega+\left(\begin{array}{cc}
0 & F_{1}^{T} \delta_{2}^{T} \\
0 & F_{2}^{T} \delta_{2}^{T}
\end{array}\right) P\left(\begin{array}{l}
\eta \\
\psi
\end{array}\right)\right]
\end{aligned}
$$

with $R$ defined as in Theorem 1. Expanding the last term in (25) as in the proof of Theorem 1, and using the estimate dynamics (16), one gets

$$
\begin{aligned}
\dot{V}= & -\omega^{T}\left(k_{d} I+\delta^{T} C \delta\right) \omega-\left(\begin{array}{ll}
\eta^{T} & \psi^{T}
\end{array}\right) R\left(\begin{array}{c}
\eta \\
\psi
\end{array}\right) \\
& +\omega^{T} D+k_{d} \omega^{T} J_{m b}^{-1} \chi_{D}-\left(\begin{array}{ll}
e_{\eta}^{T} & e_{\psi}^{T}
\end{array}\right) Q\left(\begin{array}{c}
e_{\eta} \\
e_{\psi}
\end{array}\right) \\
& +\left(\begin{array}{ll}
e_{\eta}^{T} & e_{\psi}^{T}
\end{array}\right)\left(\begin{array}{c}
B_{1} \\
B_{2}
\end{array}\right) \chi_{D}
\end{aligned}
$$

where

$$
\begin{aligned}
& B_{1}=\left(K+P_{2} C-P_{1}\right) \delta J_{m b}^{-1} \\
& B_{2}=\left[\left(C+P_{3}-P_{2}^{T}\right) \delta-k_{d} \delta J_{m b}^{-1}\right] J_{m b}^{-1} .
\end{aligned}
$$


Now note that, for a certain fixed $T>0$

$$
\begin{aligned}
&\left\|\chi_{D}\right\| \leq\left\|\mathcal{R}_{b i}\left(q_{0}, q\right) \int_{0}^{t} \mathcal{R}_{i b}\left(q_{0}(\tau), q(\tau)\right) D(\tau) d \tau\right\| \\
& \leq \int_{0}^{t}\|D(\tau)\| d \tau \leq \alpha_{1} T, \quad \forall \quad t \leq T \\
& \omega^{T} D \leq \alpha_{1}\|\omega\| \\
& {\left.\left[\begin{array}{ll}
k_{d} \omega^{T} J_{m b}^{-1}+\left(e_{\eta}^{T}\right. & e_{\psi}^{T}
\end{array}\right)\left(\begin{array}{c}
B_{1} \\
B_{2}
\end{array}\right)\right] \chi_{D} \leq \alpha_{1} \alpha_{2} T\left\|\begin{array}{l}
\omega \\
e_{\eta} \\
e_{\psi}
\end{array}\right\| \leq \alpha_{1} \alpha_{2} T\|\bar{x}\| }
\end{aligned}
$$

where

$$
\bar{x}=\left(\begin{array}{lllll}
\omega^{T} & \eta^{T} & \psi^{T} & e_{\eta}^{T} & e_{\psi}^{T}
\end{array}\right)^{T}
$$

and $\alpha_{2}=k_{d}\left\|J_{m b}^{-1}\right\|+\left\|B_{1}\right\|+\left\|B_{2}\right\|$. Hence, one finally obtains

$$
\dot{V} \leq-\lambda\|\bar{x}\|^{2}+\alpha_{1}\left(1+\alpha_{2} T\right)\|\bar{x}\|
$$

with $\lambda=\min \sigma\left(\operatorname{diag}\left\{k_{d} I+\delta^{T} C \delta, R, Q\right\}\right)$. Now, since

$$
\begin{aligned}
& \left\|\begin{array}{c}
1-q_{0} \\
q
\end{array}\right\|^{2} \leq\left\|\begin{array}{c}
1-q_{0} \\
q
\end{array}\right\| \\
& \|x\|^{2}=\left\|\begin{array}{c}
1-q_{0} \\
q \\
\bar{x}
\end{array}\right\|^{2} \leq\left\|\begin{array}{c}
1-q_{0} \\
q
\end{array}\right\|^{2}+\|\bar{x}\|^{2}
\end{aligned}
$$

one rewrites (26) as follows

$$
\begin{aligned}
\dot{V} & \leq-\lambda\|x\|^{2}+\lambda\left\|\begin{array}{c}
1-q_{0} \\
q
\end{array}\right\|+\alpha_{1}\left(1+\alpha_{2} T\right)\|\bar{x}\| \\
& \leq-\lambda\|x\|^{2}+\alpha_{1}\left(1+\alpha_{2} T\right)\|x\|
\end{aligned}
$$

for $\alpha_{1}\left(1+\alpha_{2} T\right) \geq \lambda$. This is verified for

$$
T \geq T_{1}=\frac{1}{\alpha_{2}}\left(\frac{\lambda}{\alpha_{1}}-1\right) .
$$

Hence, taking $\vartheta \in(0,1)$, one writes

$$
\begin{aligned}
\dot{V} & \leq-\lambda(1-\vartheta)\|x\|^{2}+\left(\alpha_{1}\left(1+\alpha_{2} T\right)-\vartheta \lambda\|x\|\right)\|x\| \\
& \leq-\lambda(1-\vartheta)\|x\|^{2}
\end{aligned}
$$

for $\|x\| \geq \mu=\frac{\alpha_{1}\left(1+\alpha_{2} T\right)}{\vartheta \lambda}, \quad T \geq T_{1}$.

If $b$ is the fixed ultimate bound for the trajectories, one works out [22]

$$
\|x\| \leq \sqrt{\frac{\alpha_{M}}{\alpha_{m}}} \alpha_{1}\left(1+\alpha_{2} T\right) \leq b, \quad T \geq T_{1}
$$

where it has been used the fact that $V$ is positive definite, namely $\alpha_{m}\|x\|^{2} \leq V \leq \alpha_{M}\|x\|^{2}$. One finally determine a second condition for $T$

$$
T \leq T_{2}=\frac{1}{\alpha_{2}}\left(b \sqrt{\frac{\alpha_{m}}{\alpha_{M}}} \frac{\vartheta \lambda}{\alpha_{1}}-1\right) .
$$

Note that $T_{2} \geq T_{1}$ for

$$
b \geq \frac{1}{\vartheta} \sqrt{\frac{\alpha_{M}}{\alpha_{m}}} .
$$

TABLE I

Parameters of the Flexible Dynamics

\begin{tabular}{ccc}
\hline \hline & Natural Frequency $(\mathrm{rad} / \mathrm{s})$ & Damping \\
\hline Mode 1 & 0.7681 & 0.005607 \\
Mode 2 & 1.1038 & 0.008620 \\
Mode 3 & 1.8733 & 0.01283 \\
Mode 4 & 2.5496 & 0.02516 \\
\hline
\end{tabular}

Hence $T$ must be such that $T \in\left[T_{1}, T_{2}\right]$. From (28) one gets

$$
b \geq \alpha_{1} \frac{1}{\vartheta \lambda} \sqrt{\frac{\alpha_{M}}{\alpha_{m}}}
$$

which is the condition relating the bound to the size of the perturbation, with

$$
\begin{aligned}
& \alpha_{m}=\min \left\{k_{p}, \frac{1}{2} \lambda_{\text {min }}\left(J_{m b}\right), \frac{1}{2} \lambda_{\min }(P), \frac{1}{2} \lambda_{\min }(\Gamma)\right\} \\
& \alpha_{M}=\max \left\{k_{p}, \frac{1}{2} \lambda_{\max }\left(J_{m b}\right), \frac{1}{2} \lambda_{\max }(P), \frac{1}{2} \lambda_{\max }(\Gamma)\right\}
\end{aligned}
$$

and $\lambda_{\min }(\cdot), \lambda_{\max }(\cdot)$ the lowest and greatest eigenvalue of the corresponding matrix. Note finally that if $\alpha_{1}\left(1+\alpha_{2} T\right) \leq \lambda$, namely when $T \leq T_{1}$, one obtains $\mu=1 / \vartheta$ and the condition

$$
\|x\| \leq \sqrt{\frac{\alpha_{M}}{\alpha_{m}}} \frac{1}{\vartheta} \leq b, \quad \forall \quad t \leq T \leq T_{1} .
$$

This completes the proof of Theorem 2 .

\section{SIMULATION RESULTS}

The controller (16), (17) has been applied for the control of a spacecraft with a flexible array; four bending modes have been considered for the implemented spacecraft model (see Table I), while for designing the controller only the first three bending modes have been taken into account.

The spacecraft is also characterized by a nominal main body inertia matrix

$$
J_{m b}=\left(\begin{array}{ccc}
350 & 3 & 4 \\
3 & 280 & 10 \\
4 & 10 & 190
\end{array}\right) \mathrm{Kg} / \mathrm{m}^{2}
$$

and by the coupling matrices

$$
\begin{aligned}
\delta & =\left(\begin{array}{ccc}
6.45637 & 1.27814 & 2.15629 \\
-1.25619 & 0.91756 & -1.67264 \\
1.11687 & 2.48901 & -0.83674 \\
1.23637 & -2.6581 & -1.12503
\end{array}\right) \sqrt{\mathrm{Kg}} \mathrm{m} / \mathrm{s}^{2} \\
\delta_{2} & =\left(\begin{array}{cc}
2.342552 & 10^{-2} \\
-4.225368 & 10^{-3} \\
3.912984 & 10^{-2} \\
7.026176 & 10^{-2}
\end{array}\right) \sqrt{\mathrm{Kg}} \mathrm{m} /\left(\mathrm{Vs}^{2}\right) .
\end{aligned}
$$




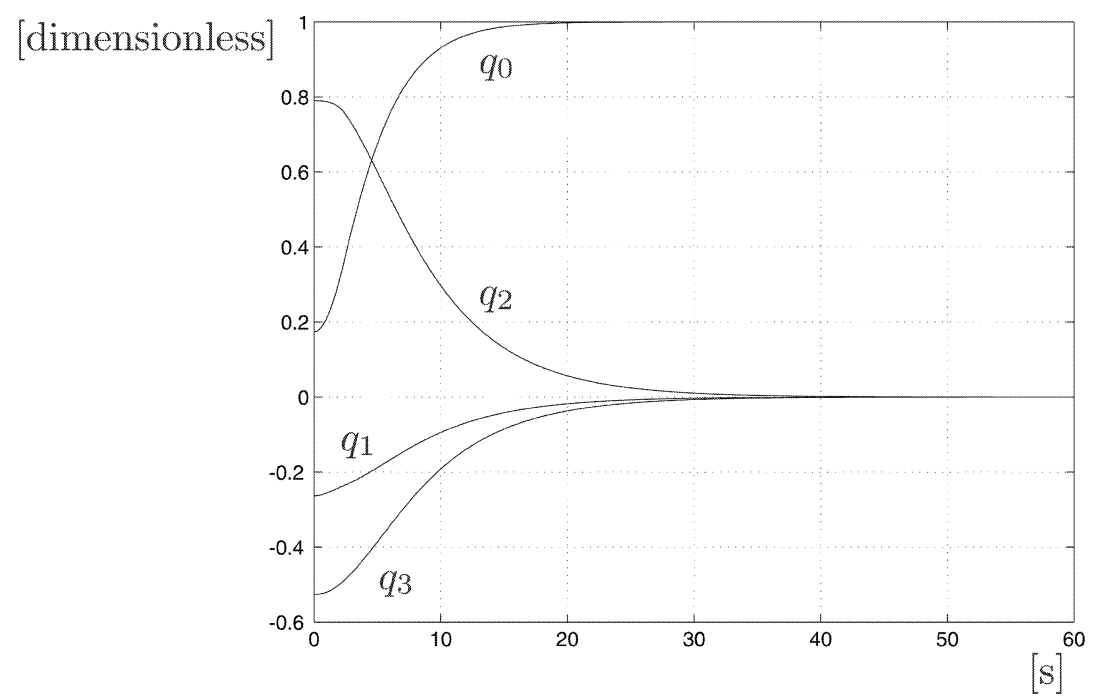

Fig. 1. Quaternions $q_{0}, q_{1} q_{2}, q_{3}$; disturbances and active control absent.

An external disturbance $D_{e}$ has been considered. In particular, here $D_{e}$ is a random disturbance torque, depending on the spacecraft attitude and given by

$$
D_{e}=D_{e, \max }\left(\begin{array}{c}
\mathcal{N}\left(q_{1}\right) \\
\mathcal{N}\left(q_{2}\right) \\
\mathcal{N}\left(q_{3}\right)
\end{array}\right)
$$

whose maximum absolute value $D_{e, \max }$ has been fixed equal to $6.15 \mathrm{Nm}[23] ; \mathcal{N}(t)$ denotes the normal distribution with mean zero and standard deviation one. This type of disturbance could be due to the environment forces acting on the spacecraft, such as the ones due to the aerodynamic drag. Moreover, an uncertainty $\Delta J$ on the inertia matrix $J$ has been considered, given by

$$
\begin{aligned}
\Delta J & =\left(\begin{array}{ccc}
4.20 & 0.900 & 0.600 \\
0.900 & -7.00 & 2.50 \\
0.600 & 2.50 & 5.89
\end{array}\right) \\
& =\left(\begin{array}{ccc}
0.012 J_{m b, 11} & 0.30 J_{m b, 12} & 0.15 J_{m b, 13} \\
0.30 J_{m b, 12} & -0.025 J_{m b, 22} & 0.25 J_{m b, 23} \\
0.15 J_{m b, 13} & 0.25 J_{m b, 23} & 0.031 J_{m b, 33}
\end{array}\right)
\end{aligned}
$$

whose elements correspond to a certain percentage of the elements of the matrix $J_{m b}$. The true main body inertia matrix is hence $J_{m b}+\Delta J$. The disturbance $D_{e}$ and this parameter uncertainty determine the term $D$ in (5), given by

$$
D=-\tilde{\omega} \Delta J \omega+D_{e}-\Delta J \dot{\omega} .
$$

The maneuver is a rotation of $160^{\circ}$; this corresponds to the following initial values for the quaternions

$$
\begin{array}{ll}
q_{0}=0.173648, & q_{1}=-0.263201 \\
q_{2}=0.789603, & q_{3}=-0.526402 .
\end{array}
$$

TABLE II

Characteristics of the Piezoelectric and Bounding Layers and of the Flexible Panel

\begin{tabular}{ccc}
\hline \hline Piezoelectric Layer & Bounding Layer & Flexible Panel \\
\hline$d_{p}=171 \quad 10^{-12} \mathrm{~m}$ & $E_{b}=1.110^{6} \mathrm{~N} / \mathrm{m}^{2}$ & $l=5 \mathrm{~m}$ \\
$E_{p}=13910^{9} \mathrm{~N} / \mathrm{m}^{2}$ & $t_{b}=2.110^{-3} \mathrm{~m}$ & $l_{a}=0.8 \mathrm{~m}$ \\
$t_{p}=2.1 \quad 10^{-3} \mathrm{~m}$ & & $l_{b}=0.1 \mathrm{~m}$ \\
& & $E=6.810^{10} \mathrm{~N} / \mathrm{m}^{2}$ \\
\hline
\end{tabular}

We suppose that the spacecraft is idle at $t=0$, i.e. the other variables are zero at the initial time. Also the estimate variable initial conditions have been chosen equal to zero. Hence, we set $\hat{\chi}_{u}(0)=0$ as initial values of the estimate $\hat{\chi}_{u}$ of $\chi_{u}$, obtained by implementing the dynamics

$$
\dot{\hat{\chi}}_{u}=-\tilde{\hat{\omega}} \hat{\chi}_{u}+u
$$

where $u$ is determined on the basis of the nominal system parameters. Clearly, $\hat{\omega}$ is given by (14) with $\hat{\chi}_{u}$ in the place of $\chi$ and with $J_{m b}$ given by the nominal value.

The actuators on the main body have a saturation at $30 \mathrm{Nm}$, while the piezoelectric actuator has a saturation at $20 \mathrm{~V}$. The characteristics of the piezoelectric are summarized by the piezoelectric charge constant $d_{p}$, the Young modulus of elasticity $E_{p}$ and the thickness $t_{p}$, reported in Table II [1-3, 6] along with the bounding layer parameters $E_{b}$ (Young modulus of elasticity), $t_{b}$ (thickness). The bending moment $M_{p}$ due to the piezoelectric films is proportional to the applied voltage according to (4) with

$$
c_{p}=d_{p} l_{a} E_{p} \frac{E_{b} t_{b}\left(t_{p}+t_{b}\right)+E l_{b}\left(t_{p}+2 t_{b}+l_{b}\right)}{2\left(E_{p} t_{p}+E_{b} t_{b}+E l_{b}\right)} \mathrm{Nm} / \mathrm{V}
$$




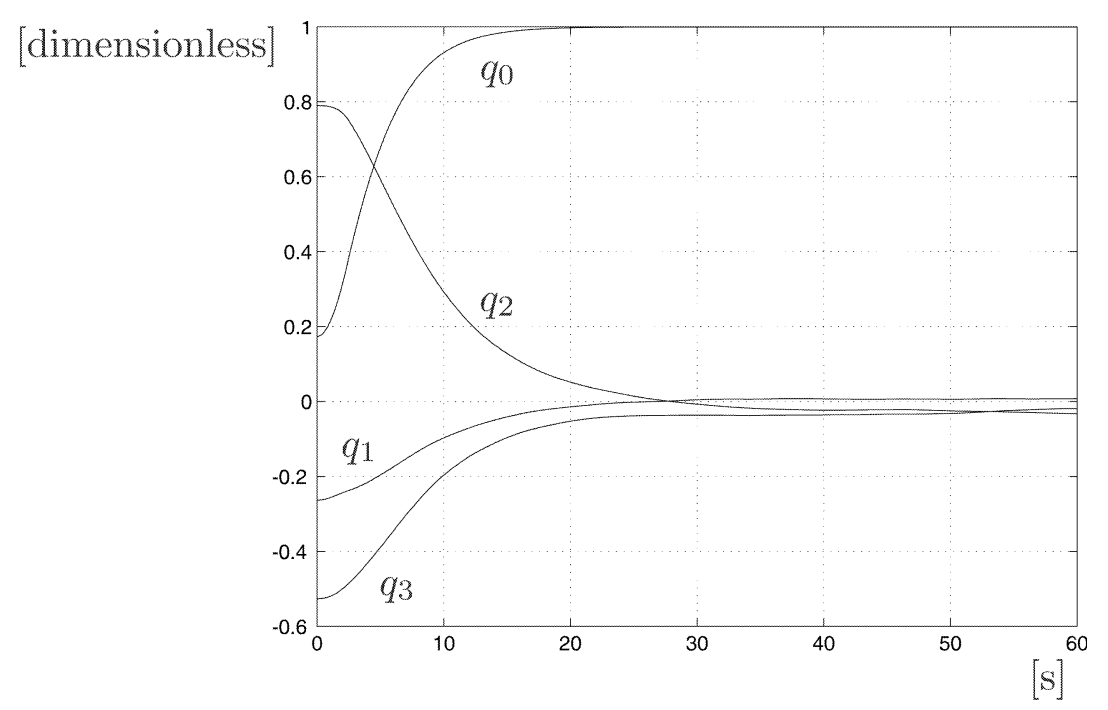

Fig. 2. Quaternions $q_{0}, q_{1} q_{2}, q_{3}$; with disturbances; active control absent.

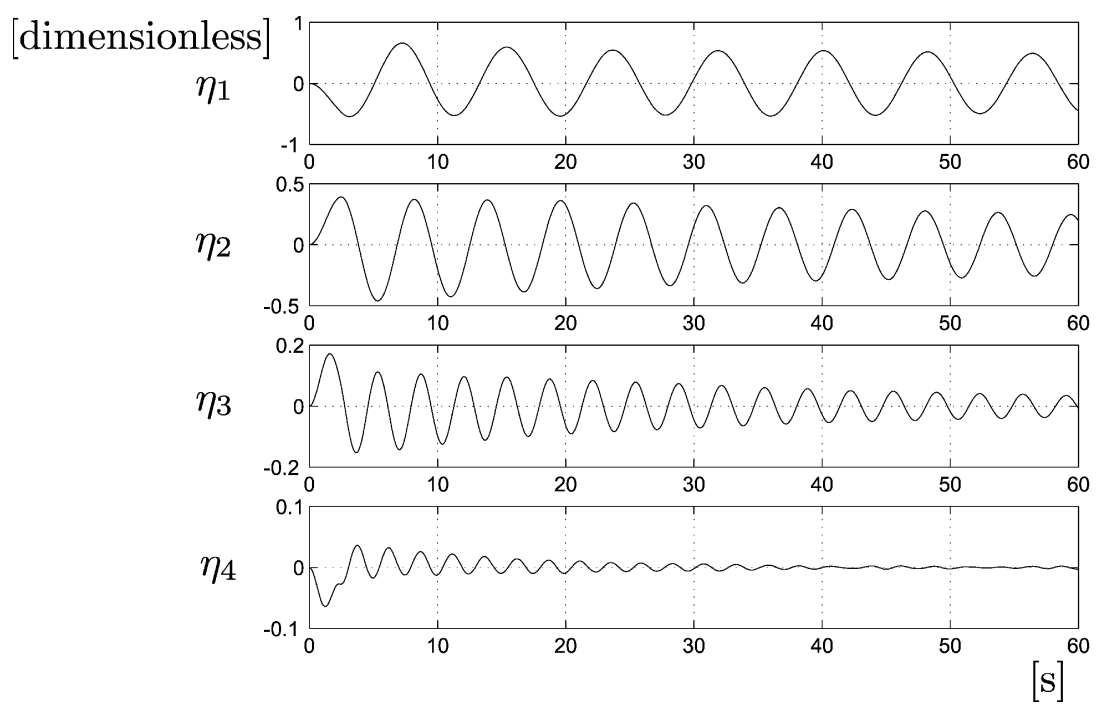

Fig. 3. Modal displacements $\eta_{1}, \eta_{2}, \eta_{3}, \eta_{4}$; with disturbances; active control absent.

The length, width and thickness of the flexible panel, constituted by aluminum, are $l, l_{a}, l_{b}$, respectively, while the Young modulus of elasticity is $E$.

The gains $k_{p}, k_{d}$ in the controller (17) have been set equal to

$$
k_{p}=1000, \quad k_{d}=3000 .
$$

These values ensure to reach the desired attitude in less than $40 \mathrm{~s}$ when the flexible elements are absent, all the state is measurable, and $D=0$ (see Fig. 1). It is easy to check that with this choice of $k_{p}, k_{d}$, the linearization of the closed-loop dynamics, obtained from (5) neglecting the flexibility and the disturbance $D$

$$
\begin{aligned}
& \dot{q}=\frac{1}{2}\left(q_{0} I+\tilde{q}\right) \omega \\
& \dot{\omega}=-J_{m b}^{-1}\left(k_{p} q+k_{d} \omega\right)-J_{m b}^{-1} \tilde{\omega} J \omega
\end{aligned}
$$

about $q_{0}=1, q=0, \dot{q}_{0}=0, \dot{q}=0$, i.e. the dynamics

$$
\left(\begin{array}{l}
\dot{q} \\
\ddot{q}
\end{array}\right)=\left(\begin{array}{cc}
0 & I \\
-\frac{k_{p}}{2} J_{m b}^{-1} & -k_{d} J_{m b}^{-1}
\end{array}\right)\left(\begin{array}{l}
q \\
\dot{q}
\end{array}\right)
$$

have eigenvalues given by

$$
\begin{array}{lll}
\lambda_{1}=-8.0248, & \lambda_{2}=-4.5559, & \lambda_{3}=-5.3154 \\
\lambda_{4}=-3.5965, & \lambda_{5}=-3.4778, & \lambda_{6}=-3.5564 .
\end{array}
$$

On the other hand, when flexible appendages are present and $D=0$, one observes an attitude error at steady-state (see Fig. 2) due to (the disturbance $D$ and) the poor behavior of the flexible dynamics (Fig. 3). This behavior can be improved by exploiting the contribution due to piezoelectric actuators bonded to the panel. The matrices $F_{1}, F_{2}$ are designed so that the eigenvalues of the dynamics of the first three 


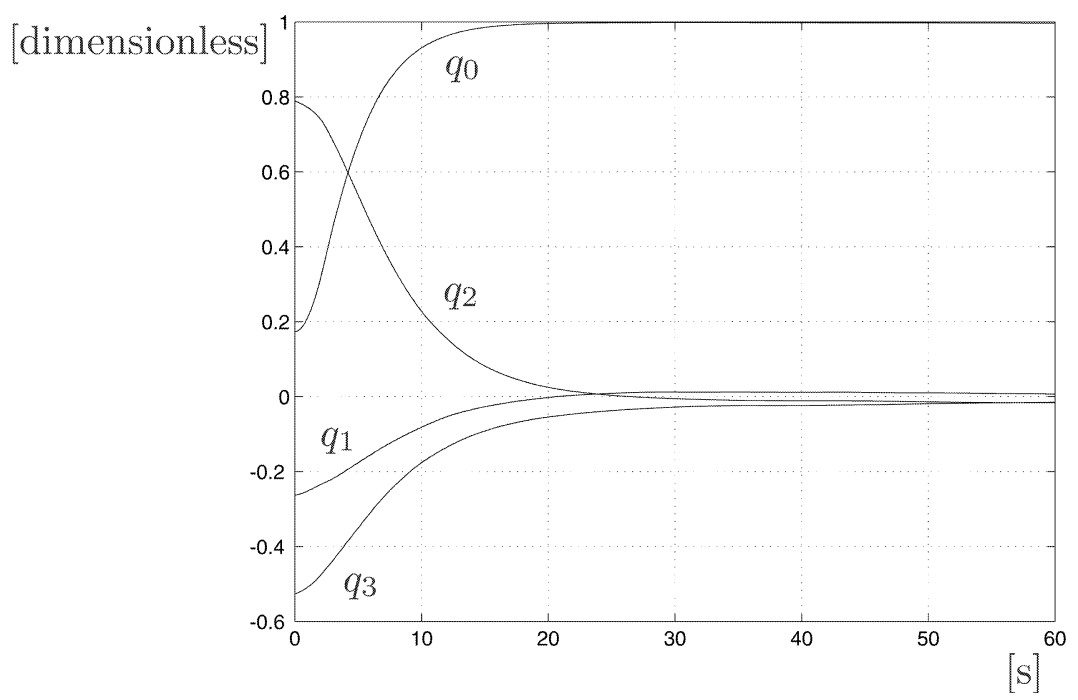

Fig. 4. Quaternions $q_{0}, q_{1} q_{2}, q_{3}$; with disturbances and active control.

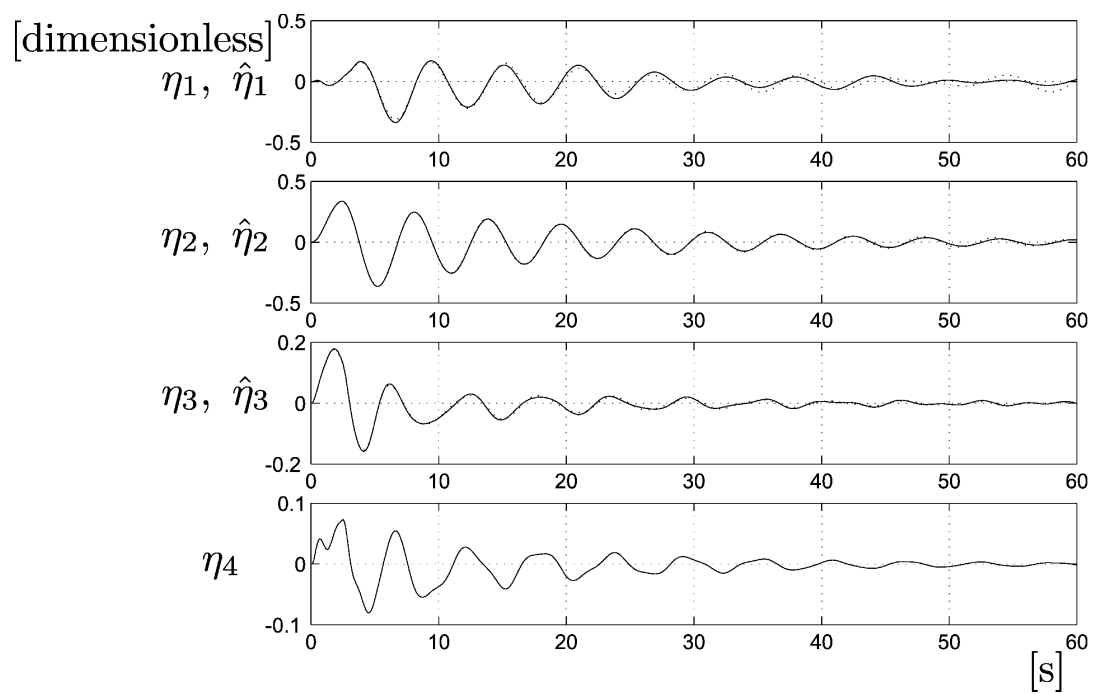

Fig. 5. Modal displacements $\eta_{1}, \eta_{2}, \eta_{3}, \eta_{4}$ and estimates $\hat{\eta}_{1}, \hat{\eta}_{2}, \hat{\eta}_{3}$; with disturbances and active control.

modes have natural frequencies

$$
\omega_{0}=\left(\begin{array}{c}
1.0973 \\
1.8213 \\
5.6199
\end{array}\right) \mathrm{rad} / \mathrm{s}
$$

while for the damping

$$
\zeta_{0}=\left(\begin{array}{c}
0.05 \\
0.2 \\
0.4
\end{array}\right) .
$$

Therefore, one has

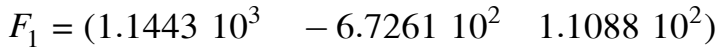

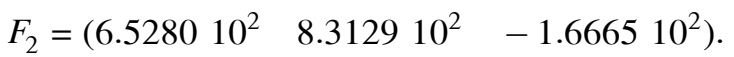

In Fig. 4 it is shown how the attitude error is decreased thanks to a better modal behavior (Fig. 5). Note in particular that the estimates $\hat{\eta}_{1}, \hat{\eta}_{2}, \hat{\eta}_{3}$ converge to the real values of $\eta_{1}, \eta_{2}, \eta_{3}$. Observe also in Fig. 5 that the fourth mode remains stable, even if its behavior is worse than before due to the coupling with the other modal dynamics induced by the feedback. Moreover, in Fig. 6 the angular velocity components $\omega_{i}$ and their estimates $\hat{\omega}_{i}, i=1,2,3$, are shown, while in Fig. 7 the input components $u_{i}$, $i=1,2,3$, and $u_{p}$ are given. Finally, in Fig. 8 the values of the components $D_{i}$ of $D$ are shown.

In particular, note that, according to the results of Section III, the proposed controller still ensures that the state variables are bounded in this case in which $D \neq 0$. This is clearly shown by the quaternion behavior in Fig. 5, which remains acceptable; note that in Fig. 5 the final values of $q_{0}, \ldots, q_{3}$ are about

$$
\begin{aligned}
& q_{0} \simeq 0.997, \quad q_{1} \simeq 0.0693 \\
& q_{2} \simeq 0.0159, \quad q_{3} \simeq-0.0148 .
\end{aligned}
$$



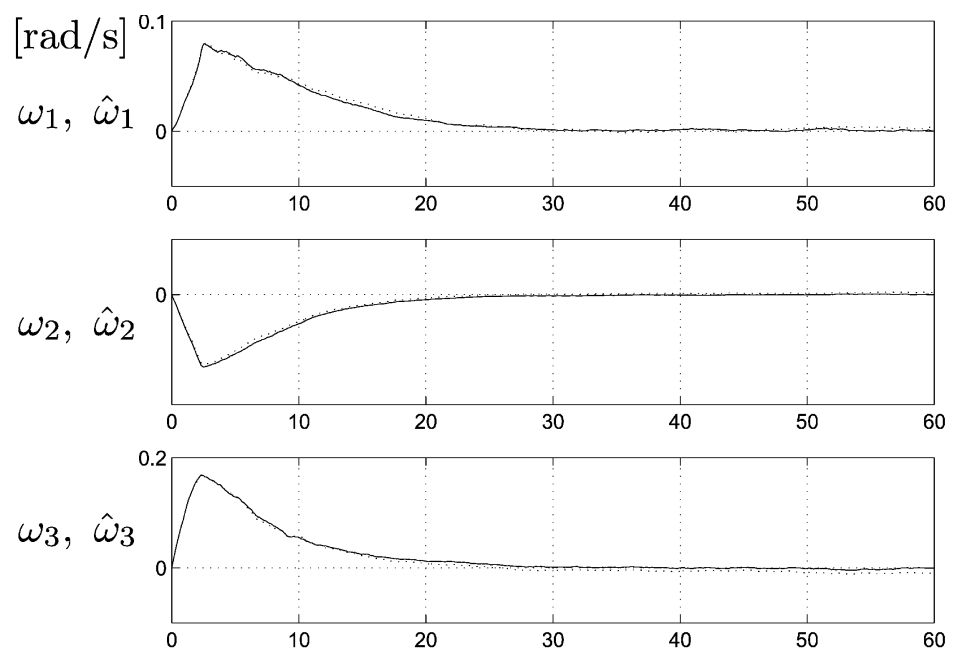

$[\mathrm{S}]$

Fig. 6. Angular velocities $\omega_{1}, \omega_{2}, \omega_{3}$ and estimates $\hat{\omega}_{1}, \hat{\omega}_{2}, \hat{\omega}_{3}$; with disturbances and active control.
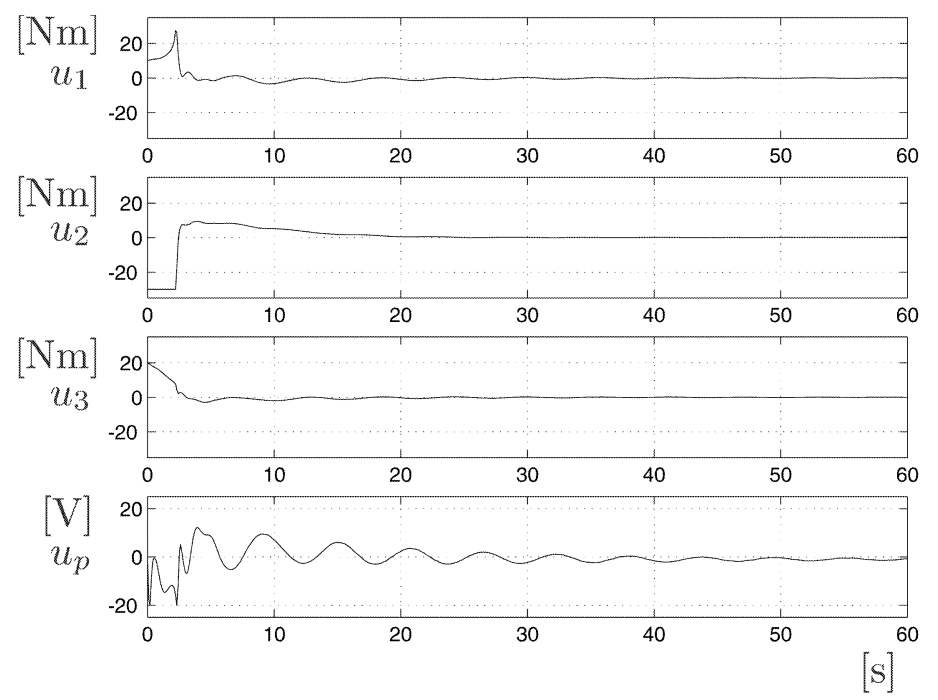

Fig. 7. Control inputs $u_{1} u_{2}, u_{3}$ and $u_{p}$; with disturbances and active control.
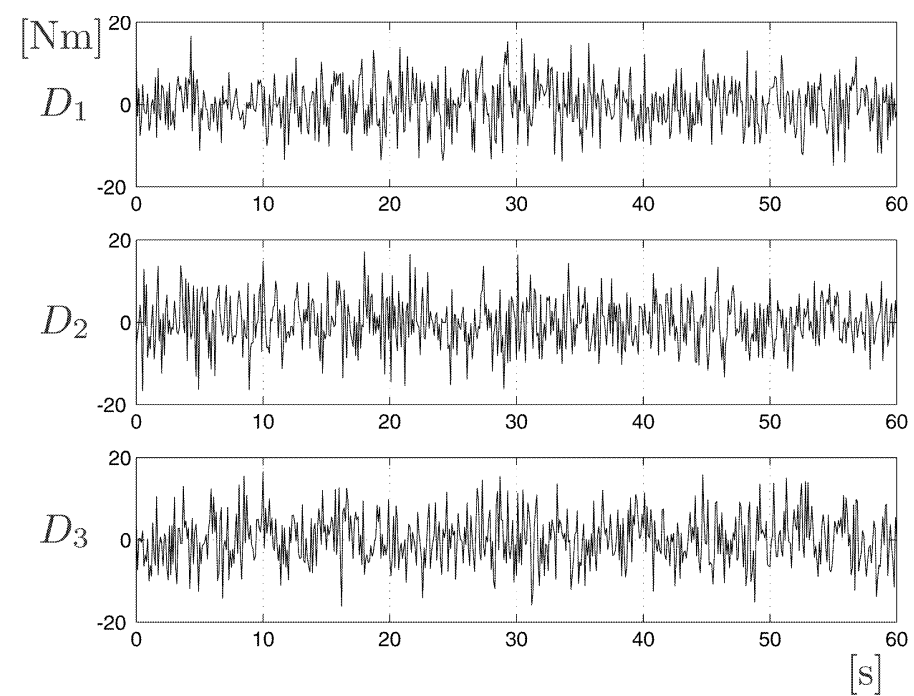

Fig. 8. Disturbance components $D_{1}, D_{2}, D_{3}$. 
Clearly, this implies that the angle $\Phi$, denoting not only the rotation angle but also the attitude error angle, goes to a value different from zero, due to the presence of the disturbance. Hence, the disturbance and the parameter variations do not impair the stability of the control scheme, and the proposed dynamic controller possesses some robustness properties.

\section{CONCLUSIONS}

In this work a dynamic controller has been presented for rest-to-rest spacecraft maneuvers. Only the attitude variables are measured; this is a clear advantage over classic control schemes. The active vibration suppression, performed by piezoelectric actuators, is particularly effective. In fact, they have been proved useful in eliminating the degradation of the control law due to the use of state estimates, disturbances and parameter variations. The main drawback in their application in spacecraft missions remains the considerable energy consumption. However, they represent an interesting example of integration between distributed actuators and structures.

\section{ACKNOWLEDGMENTS}

The author thanks Salvatore Monaco for the helpful discussions and the technical suggestions.

\section{REFERENCES}

[1] Baz, A., and Poh, S. (1988)

Performance of an active control system with piezoelectric actuators.

Journal of Sound and Vibration, 126 (Feb. 1988), 327-343.

[2] Burke, S. E., and Hubbard, J. E., Jr. (1988)

Distributed actuator control design for flexible beams. Automatica, 24 (May 1988), 619-627.

[3] Li, Z., and Bainum, P. M. (1994)

Vibration control of flexible spacecraft integrating a momentum exchange controller and a distributed piezoelectric actuator.

Journal of Sound and Vibration, 177 (Apr. 1994), 539-553.

[4] Bailey, T., and Hubbard, J. E., Jr. (1985)

Distributed piezoelectric-polymer active vibration control of a cantilever beam.

Journal of Guidance, 8 (May 1985), 605-611.

[5] Hyland, D. C., Junkins, J. L., and Longman, R. W. (1993) Active control technology for large space structures. AIAA Journal of Guidance, Control, and Dynamics, 16 (May 1993), 801-821.

[6] Di Gennaro, S. (1998)

Active vibration suppression in flexible spacecraft attitude tracking.

Journal of Guidance, Control, and Dynamics, 21 (Mar. 1998), 400-408.

[7] Dwyer, T. A. W. (1984)

Exact nonlinear control of large angle rotational maneuvers.

IEEE Transactions on Automatic Control, AC-29 (Sept. 1984), 769-774.
[8] Monaco, S., and Stornelli, S. (1985)

A nonlinear feedback control law for attitude control. In M. Hazewinkel and M. Fliess (Eds.), Algebraic and Geometric Methods in Nonlinear Control Theory, Dordrecht, Holland: Reidel, 1985, 573-595.

[9] Monaco, S., and Stornelli, S. (1985)

A nonlinear attitude control law for a satellite with flexible appendages.

In Proceedings of the 24th Conference on Decision and Control, Ft. Lauderdale, FL, 1985, 1654-1659.

[10] Monaco, S., Normand-Cyrot, D., and Stornelli, S. (1986) Sampled nonlinear control for large angle maneuvers of flexible spacecraft.

In Proceedings of the 2nd International Symposium on Spacecraft Flight Dynamics, ESA SP-255, Darmstadt, Germany, 1986, 31-38.

[11] Dwyer, T. A. W., Sira-Ramirez, H., Monaco, S., and Stornelli, S. (1987)

Variable structure control of globally feedback decoupled deformable vehicle maneuvers.

In Proceedings of the 27th Conference on Decision and Control, Los Angeles, CA, 1987, 1281-1287.

[12] Georgiou, G., Di Gennaro, S., Monaco, S., and

Normand-Cyrot, D. (1991)

On the nonlinear adaptive control of a flexible spacecraft. In Proceedings of the 1st ESA Conference on 'Spacecraft Guidance, Navigation and Control Systems', ESA SP-323, Noordwijk, The Netherlands, 1991, 509-514.

[13] Di Gennaro, S., Monaco, S., Normand-Cyrot, D., and Pignatelli, A. (1996)

Digital controllers for attitude manoeuvring: Experimental results.

In Proceedings of the 2nd International Symposium on Spacecraft Flight Dynamics, ESA SP-381, Noordwijk, The Netherlands, 1996, 439-446.

[14] Di Gennaro, S., Monaco, S., and Normand-Cyrot, D. (1999) Nonlinear digital scheme for attitude tracking.

AIAA Journal of Guidance, Control, and Dynamics, 22, 3 (1999), 467-477.

[15] Wie, B., and Barba, P. M. (1985)

Quaternion feedback for spacecraft large angle maneuvers.

AIAA Journal of Guidance, 8 (Mar. 1985), 360-365.

[16] Di Gennaro, S. (2003)

Passive attitude control of flexible spacecraft from quaternion measures.

Journal of Optimization Theory and Applications, 116 (Jan. 2003).

[17] Di Gennaro, S. (2002)

Attitude tracking for flexible spacecraft from quaternion measures.

Presented at the 41st IEEE Conference on Decision and Control, Las Vegas, NV, 2002.

[18] Ickes, B. P. (1970)

A new method for performing digital control system attitude computations using quaternions.

AIAA Journal, 8 (Jan. 1970), 13-17.

[19] Wertz, J. R. (Ed.) (1978)

Spacecraft Attitude Determination and Control.

Dordrecht, Holland: Kluwer Academic Publishers, 1978.

[20] Di Gennaro, S. (1998)

Adaptive robust stabilization of spacecraft in presence of disturbances.

Journal of Optimization Theory and Applications, 98 (Mar. 1998), 545-568.

[21] Di Gennaro, S. (1996)

Output feedback stabilization of flexible spacecraft. In Proceedings of the 35th Conference on Decision and Control, Kobe, Japan, 1996, 497-502. 
Stefano Di Gennaro obtained the degree in nuclear engineering in 1987 (summa cum laude), and the Ph.D. degree in system engineering in 1992, both from the University of Rome "La Sapienza," Rome, Italy.

In October 1990 he joined the Department of Electrical Engineering, University of L'Aquila, as assistant professor of Automatic Control. He has been associate professor of Automatic Control since 2002. In 1986 he was visiting scientist at the Nuclear Research Center "ENEA-Casaccia." From 1991 to 1998 he was visiting professor at the Laboratoire des Signaux et Systèmes, CNRS-Paris, and of the Department of Electrical Engineering of Princeton University, Princeton, NJ, USA, from 1993 to 1995. From 1993 to the present he has been visiting professor of the Centro de Investigacion y Estudios Avanzados del IPN, Unidad Ciudad de Mexico and Unidad Guadalajara, Mexico. In 1998 he visited the Department of Electrical Engineering and Computer Science, University of California at Berkeley. He is working in the area of nonlinear control applied to spacecraft attitude control, electric machines, nonlinear regulation, and in the area of control of hybrid systems. 